\title{
EFFICIENCY IN NUCLEAR FUELING
}

\section{NOGUCHI}

\author{
Astronomical Institute, Tohoku University \\ Aoba, Sendai 980-77, Japan
}

Starburst phenomena in interacting and merging galaxies have been one of the most widely investigated subjects in today's galactic astronomy. On the theoretical side, a large body of numerical studies have been performed in order to interpret available observational data. Numerical simulations have been advanced to the point where they can include interstellar medium (ISM) and star formation process.

Most numerical simulations try to model the interstellar matter as a continuous fluid and use the Smoothed Particle Hydrodynamics (SPH) as the numerical code. Although the SPH seems to be fully tested from the numerical point of view, use of it can not necessarily be justified, considering highy clumpy nature of the actual ISM, most part of which is occupied by massive and discrete molecular or neutral hydrogen clouds.

I have used another description of the ISM, namely, the so-called stickyparticle model and investigated how the starburst characteristics depend on the parameters specifying the gas cloud system. In the sticky-particle model, the ISM is modelled as a system comprising numerous discrete gas clouds, which dissipate kinetic energy is mutual inelastic collisions. One of the most important physical parameters is the timescale of cloud-cloud collisions, $\tau_{c o l}$, which is in turn determined by a combination of several fundamental parameters such as the cloud radius, the cloud velocity dispersion, and the cloud number density.

In the models presented here (Fig.1), $\tau_{\text {col }}$ has been varied between $2 \times 10^{7}$ yr and $2 \times 10^{9} \mathrm{yr}$, which brackets observationally inferred values for our Galaxy. As Fig.1 indicates, the maximum strength of the starburst and the epoch of the maximum depend on the cloud-cloud collisional timescale strongly. As $\tau_{c o l}$ increases, the peak star formation rate decreases and the epoch of the peak activity is delayed. The model with $\tau_{c o l}=2 \times 10^{7} \mathrm{yr}$ (dotted line) shows peak star formation well before the two galaxies merge, whereas the model with $\tau_{c o l}=2 \times 10^{8} \mathrm{yr}$ (solid line) reaches the maximum 


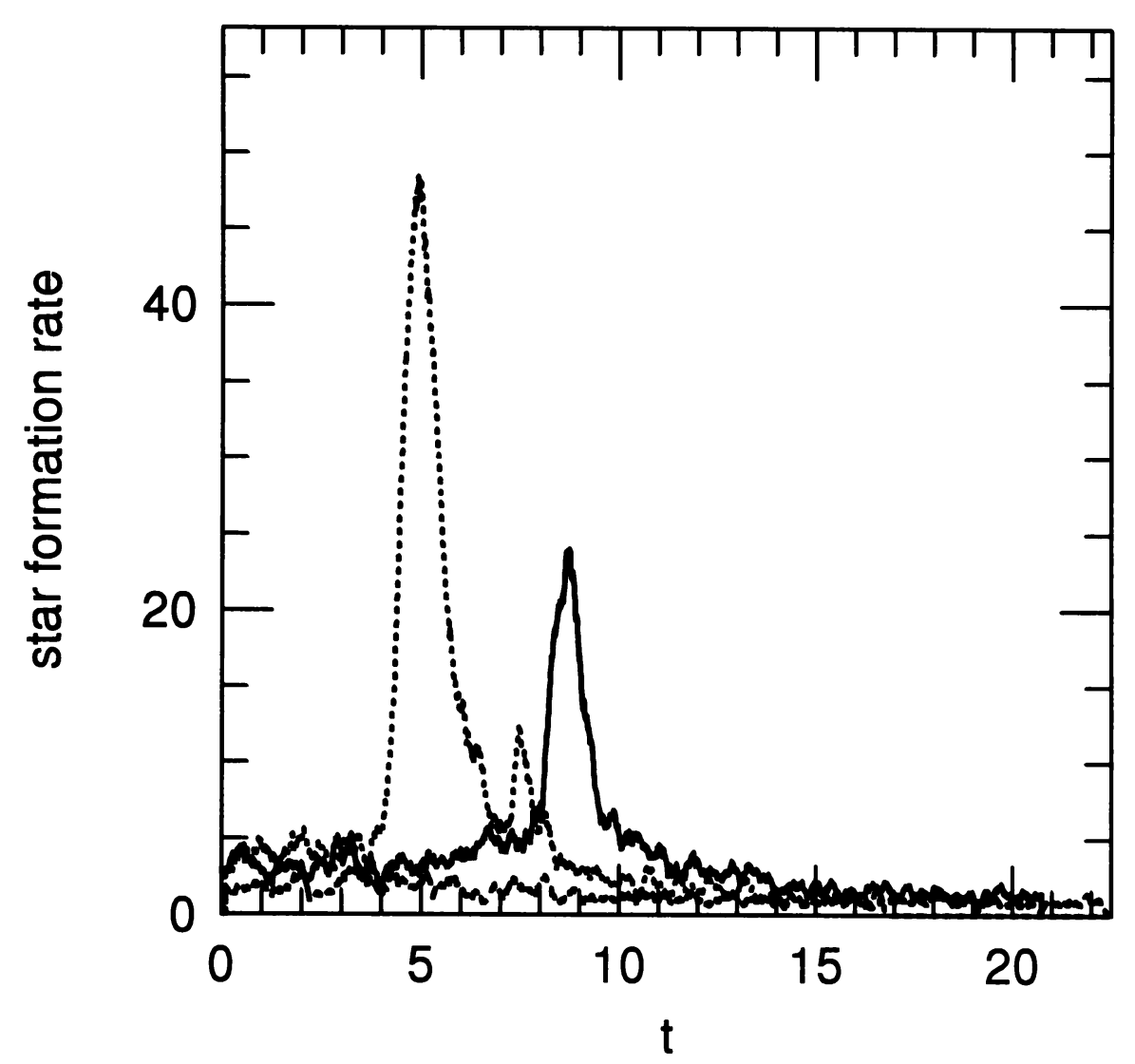

Figure 1. Star formation history in the three parabolic-and-prograde merger models between two gas-rich disk galaxies. The models are the same except for the radius of individual gas clouds. Different cloud sizes lead to different cloud-cloud collision timescales $\left(\tau_{c o l}=2 \times 10^{7}, 2 \times 10^{8}, 2 \times 10^{9}\right.$ yr for dotted, solid, and dashed lines, respectively $)$. The epoch of merger (i.e., the epoch when two galaxy nuclei coalesce into one) is $t \sim 7.5$ in all the models. One time unit is rougly $10^{8} \mathrm{yr}$, and the star formation rate is given in units of $M_{\odot} y r^{-1}$.

starburst just after the merger is completed. A continuous gas corresponds to the case in which $\tau_{c o l}$ is infinitesimally small. Therefore, a stronger and earlier starburst is expected in a SPH simulation than in a sticky-particle simulation, provided other model parameters are identical.

The results presented here indicate clearly that the starbursts triggered in galaxy mergers can have different characteristics depending on the smallscale structure of the interstellar mediun contained in the merging galaxies. 